\title{
BMJ Open Chinese herbal medicine for postpartum constipation: a protocol of systematic review and meta-analysis
}

\author{
Jingbo Zhai, ${ }^{1}$ Yan Li, ${ }^{2}$ Jingyi Lin, ${ }^{1}$ Shuo Dong, ${ }^{1}$ Jinhua $S i,{ }^{3}$ Junhua Zhang ${ }^{1}$
}

To cite: Zhai J, Li Y, Lin J, et al. Chinese herbal medicine for postpartum constipation: a protocol of systematic review and meta-analysis. BMJ Open 2019;9:e023941. doi:10.1136/ bmjopen-2018-023941

- Prepublication history and additional material for this paper are available online. To view these files, please visit the journal online (http://dx.doi. org/10.1136/bmjopen-2018023941).

$\mathrm{JZ}$ and $\mathrm{YL}$ contributed equally.

Received 2 May 2018

Revised 20 November 2018

Accepted 23 November 2018

Check for updates

(C) Author(s) (or their employer(s)) 2019. Re-use permitted under CC BY-NC. No commercial re-use. See rights and permissions. Published by BMJ.

${ }^{1}$ Institute of Traditional Chinese Medicine, Tianjin University of Traditional Chinese Medicine, Tianjin, China

${ }^{2}$ School of Nursing, Tianjin University of Traditional Chinese Medicine, Tianjin, China

${ }^{3}$ Library, Tianjin University of Traditional Chinese Medicine, Tianjin, China

Correspondence to Professor Jinhua Si; sjh665@163.com and Dr Junhua Zhang; zjhtcm@foxmail.com

\section{ABSTRACT}

Introduction Constipation is one of the most common gastrointestinal symptoms in postpartum mothers. The choice of treatments for postpartum constipation remains a challenging clinical problem. Chinese herbal medicine has become increasingly popular as an alternative therapy for constipation. This systematic review aims to evaluate the efficacy and safety of Chinese herbal medicine for postpartum constipation.

Methods and analysis We will search PubMed (1946 to present), EMBASE (1974 to present), Cochrane Central Register of Controlled Trials (all years), Web of Science (1900 to present), Chinese Biomedical Literatures Database (1978 to present), China National Knowledge Infrastructure (1979 to present) and WANFANG data (1998 to present) to identify any eligible study. No restriction will be put on the language, publication date or status of the study. The primary outcome will be the spontaneous bowel movement. Secondary outcomes will be stool consistency, quality of life, transit time, relief of constipation symptoms and adverse events. We will perform the meta-analysis when more than one trial examines the same intervention and outcomes with comparable methods in similar populations. If the heterogeneity is not significant statistically ( $p>0.10$ or $\mathrm{I}^{2}<50 \%$ ), the fixed-effect model will be built to estimate the overall intervention effects. Otherwise, the random-effect model will be used to provide more conservative results.

Ethics and dissemination No ethical issues are foreseen because no primary data will be collected. The results will be published in a peer-reviewed scientific journal.

PROSPERO registration number CRD42018093741

\section{INTRODUCTION}

Constipation is one of the most common gastrointestinal symptoms in postpartum mothers. ${ }^{1}$ A prospective study showed that the prevalence of constipation was $24 \%$ with $95 \%$ CI $13 \%$ to $36 \%$ at 3 months post partum in the USA. ${ }^{2}$ A survey found that $25 \%$ and $11.6 \%$ of women suffered from constipation at 3 and 12 months post partum in China, respectively. ${ }^{3}$

The aetiology of postpartum constipation is multifactorial. The mode of delivery and pelvic floor injury may largely contribute to defecation disorders during the postpartum
Strengths and limitations of this study

This study will only consider parallel-group randomised controlled trials to provide unbiased estimates of treatment effects.

- No language or publication date will be restricted.

- The robustness of the pooled effects will be investigated by the sensitivity analysis.

- The extreme worst-case and best-case analysis will be used to assess the potential impact of the missing data.

- A large degree of heterogeneity in terms of methodological quality and outcome measures will likely pose challenges for study comparisons.

period. ${ }^{4}$ Local trauma could be responsible for the anal sphincter spasm. ${ }^{5}$ Furthermore, taking painkillers, a lack of adequate dietary fibre, vegetable, fruit and water, and irregular meals due to baby care as well as many other situations could also lead to constipation. ${ }^{6}$ Obviously, some factors account for both functional constipation in adults and postpartum constipation. Others are only associated with postpartum constipation.

Postpartum constipation can lead to abdominal distension, abdominal pain, insomnia, inappetence and so forth. ${ }^{7}$ These symptoms have negative impacts on postpartum recovery, breast feeding, newborn health and so forth. ${ }^{8}$

Conventional therapies for constipation include stool softener, prokinetic agent, osmotic and stimulant laxative, dietary manipulation, and so forth. ${ }^{9}$ They may be associated with unexpected side effects, such as bloating, dehydration, a high recurrence rate after ceasing drugs and abdominal pain. ${ }^{10}$ According to clinical guidelines, no clinical recommendations have be provided for the management of postpartum constipation. ${ }^{81112}$ The choice of treatments for postpartum constipation remains a challenging clinical problem. 
Chinese herbal medicine (CHM) is defined as a preparation derived from plants or parts of plants. ${ }^{13} 14 \mathrm{CHM}$ includes a single herb or complex formula consisting of herbal ingredients. ${ }^{15}$ The forms of CHM include tablet, pill, decoction, oral liquid, powder, injection liquid and so forth. ${ }^{16}$

CHM has become increasingly popular as an alternative therapy for constipation. A randomised double-blind trial showed that a hemp seed pill significantly increased the responder rate in complete spontaneous bowel movement (SBM) when compared with placebo. ${ }^{17} \mathrm{~A}$ multicentre randomised controlled trial (RCT) found that a CHM decoction had a beneficial effect on reducing the Cleveland Constipation Score and improving quality of life. $^{18}$

A 2009 systematic review examined the effectiveness of CHM interventions for functional constipation. ${ }^{19}$ It showed that CHM was effective for functional constipation. However, no studies associated with postpartum constipation were included. Whether the evidence is transferable to women diagnosed with postpartum constipation remains unclear.

Many clinical trials found that CHM was beneficial for the management of postpartum constipation. For example, a clinical trial found that Xiaoyao powder significantly increased the effective rate when compared with polyethylene glycol. ${ }^{20}$ Another trial suggested that a CHM enema treatment was more effective for relieving constipation symptoms of postpartum mothers than glycerine enema. $^{21}$

A 2014 Cochrane systematic review assessed the efficacy and safety of interventions for treating postpartum constipation. ${ }^{22}$ Because of strict criteria, no eligible RCTs were included. Unfortunately, the potentially eligible studies from China could be missed as no Chinese medical databases were searched. And, it has not been updated so far.

To sum up, the evidence of the efficacy and safety of CHM for postpartum constipation still remains inconclusive due to the lack of well-performed systematic reviews on this topic.

This systematic review aims to evaluate the efficacy and safety of CHM for postpartum constipation.

\section{METHODS}

This protocol adheres to the Preferred Reporting Items for Systematic Reviews and Meta-Analyses Protocols (PRISMA) 2015. ${ }^{23}$

\section{INCLUSION CRITERIA}

\section{Types of studies}

Parallel-group RCTs will be included. No restriction will be put on the language, publication date or status of the study.

\section{Types of participants}

Women with constipation during the postpartum period will be included regardless of age, race, nationality, history of prenatal constipation, frequency of delivery, mode of delivery (vaginal delivery or caesarean section), gastrointestinal diseases and so forth.

The postpartum period ranges from an hour after the delivery of placenta to 6 weeks. ${ }^{8}$

Participants should be clinically diagnosed with constipation according to the Rome II or III diagnostic criteria, Bristol stool form scale, clinical guidelines or defined by trialists. The Rome II criteria for constipation should include at least two of the following symptoms lasting for 12 weeks or more over the period of a year: (1) Straining with more than $25 \%$ of defecations, (2) Hard stool with more than $25 \%$ of defecations, (3) Feeling of incomplete evacuation with more than $25 \%$ of defecations, (4) Sensation of anorectal obstruction with more than $25 \%$ of defecations, (5) Manual manoeuvres to facilitate more than 25\% of defecations, (6) Fewer than three bowel movements per week and (7) Insufficient criteria for irritable bowel syndrome. ${ }^{24}$ The Rome III criteria for functional constipation should include two or more of the following: (1) Straining during defecation for at least $25 \%$ of bowel movements, (2) Lumpy or hard stools in at least $25 \%$ of defecations, (3) Sensation of incomplete evacuation for at least $25 \%$ of defecations, (4) Sensation of anorectal obstruction/blockage for at least $25 \%$ of defecations, (5) Manual manoeuvres to facilitate at least $25 \%$ of defecations, (6) Fewer than three defecations per week, (7) Loose stools are rarely present without the use of laxatives and (8) There are insufficient criteria for irritable bowel syndrome. ${ }^{25}$ These symptoms should start for at least 6 months prior to diagnosis and be present for the past 3 months. ${ }^{25}$

\section{TYPES OF INTERVENTIONS}

\section{Experimental interventions}

The experimental interventions include a CHM alone and a combination of CHM and another active treatment (pharmacological or non-pharmacological intervention). Any CHM preparation (such as decoction, granula, ointment and capsule) will be considered.

\section{Comparator interventions}

The control interventions include no treatment, placebo and another active treatment. The route of delivery (such as oral and enema), dosage, frequency and duration will not be restricted.

The following comparisons will be considered if available:

1. CHM alone versus no treatment.

2. CHM alone versus placebo.

3. CHM alone versus another active treatment.

4. CHM plus another active treatment versus another active treatment alone. 
5. CHM plus another active treatment versus placebo plus another active treatment.

\section{TYPES OF OUTCOME MEASURES \\ Primary outcomes}

The primary outcome is SBM. We will consider the incidence and frequency of SBM in 24 hours or per week, the mean number or the change of SBM per week from baseline. ${ }^{1026-28}$

\section{Secondary outcomes}

Secondary outcomes include stool consistency (measured by Bristol Stool Form Scale or other tools), proportion of patients using rescue medication (such as laxatives, rectal evacuants), quality of life (measured by Maternal Postpartum Quality of Life Questionnaire or other tools), transit time (the time from the first perception of wanting to defaecate to the finish of defaecation), relief of constipation symptoms (such as sensation of straining, bloating, abdominal pain). ${ }^{26} 2930$ We will also consider other outcomes reported by the investigators when possible.

Any adverse event of the intervention on both the mother and baby (such as influence of milk production, milk rejection, etc) will be extracted and the incidence will be estimated if possible.

\section{SEARCH METHODS FOR IDENTIFICATION OF STUDIES Electronic searches}

We will search PubMed (1966 to present), EMBASE (1974 to present), Cochrane Central Register of Controlled Trials (all years), Web of Science (1900 to present), Chinese Biomedical Literatures Database (1978 to present), China National Knowledge Infrastructure (1979 to present) and WANFANG data (1998 to present) to identify any eligible study.

The search strategy is developed by a senior librarian (JS) based on previous systematic reviews. ${ }^{19} 22$ The detailed search strategy is available at online supplementary appendix 1 . The terms will be modified for other databases if necessary. No language, publication date or status will be restricted.

\section{Searching other resources}

Reference lists of primary studies and relevant reviews will be manually searched to identify additional references.

We will also conduct a search on the website of ClinicalTrials.gov, WHO International Clinical Trials Registry platform and Chinese Clinical Trial Registry to identify additional ongoing or unpublished studies.

\section{DATA COLLECTION AND ANALYSIS \\ Selection of studies}

Two review authors (JiZ and YL) will independently run search strategy to identify potentially eligible studies. The results of the literature searches will be input to the
EndNote V.X7 software. Duplicates will be omitted by using the EndNote.

The irrelevant studies will be removed by scanning titles and abstracts of references identified by the literature searches according to the inclusion criteria. Then fulltext articles will be screened to identify eligible studies. A PRISMA diagram will be used to illustrate the selection process. ${ }^{31}$ Any disagreement will be resolved through consensus or discussion with a third reviewer (JuZ).

\section{Data extraction and management}

A predetermined form will be used to extract data. The pilot test will be conducted to ensure consistency before performing the review. Two reviewers (JL and SD) will independently extract the following information:

1. General information (title, first author, year of publication, funding).

2. Study characteristics (design, randomisation, allocation, blinding, inclusion and exclusion criteria, sample size).

3. Participant characteristics (age, ethnicity, diagnosis criteria, number in each group, history of prenatal constipation, frequency of delivery, mode of delivery).

4. Intervention characteristics (experimental intervention, comparator intervention, route of delivery, dosage, frequency and duration).

5. Outcomes (primary and secondary outcomes, time points, methods of outcome assessments, blinding of outcome assessment, adverse events).

If necessary, we will contact authors of the studies included for providing further details or clarification.

\section{Assessment of risk of bias in included studies}

Two reviewers (JiZ and YL) will independently conduct the risk of bias assessment of included references using the Cochrane 'risk of bias' tool. ${ }^{32}$ The following seven domains will be assessed: random sequence generation, allocation concealment, blinding of participants and personnel, blinding of outcome assessment, incomplete outcome data, selective outcome reporting and other potential sources of bias. ${ }^{32}$ The risk of bias for each domain will be graded as low, high or unclear for each included study. ${ }^{32}$ If a study described that it was a RCT without reporting randomisation method, we will contact authors for providing further details or clarification whenever possible. If the information about the sequence generation process is insufficient to permit judgement of 'low risk' or 'high risk', this study will still be included in this systematic review and the risk of selection bias will be graded as 'unclear'.

The overall risk of bias of a study will be estimated low only if all seven domains are rated to be at low risk of bias. Otherwise, the overall risk of bias for the study is high. We will summarise the results of the risk of bias assessments with a 'risk of bias graph' and 'risk of bias summary' figure. Any disagreement will be resolved by discussion or involving a third reviewer (JuZ). 


\section{Measures of treatment effect}

For the continuous outcomes, we will calculate the mean differences (MDs) with $95 \%$ CIs. ${ }^{32}$ If the same outcome is measured using different scales, the standardised MD with $95 \%$ CI will be used to express intervention effects. ${ }^{32}$ Risk ratio with $95 \%$ CI will be used to present results for dichotomous outcomes. ${ }^{32}$

\section{Dealing with missing data}

We will contact original authors for requesting the missing data if possible. Only available data will be included in the primary analysis. However, extreme worst-case and bestcase analysis will be used to assess the potential impact of the missing data in sensitivity analysis. ${ }^{33}$

\section{Assessment of heterogeneity}

Statistical heterogeneity across the studies included will be tested using $\chi^{2}$ test and $\mathrm{I}^{2}$ statistic. The heterogeneity is significant statistically when the $\mathrm{p}$ value based on $\chi^{2}$ test less than 0.10 or $\mathrm{I}^{2}$ more than $50 \%{ }^{3435}$ If so, exploratory sensitivity or subgroup analyses will be performed to identify possible reasons. ${ }^{36}$

\section{Assessment of reporting biases}

The reporting bias will be investigated using visual funnel plots if more than 10 RCTs are included in a meta-analysis. ${ }^{32}$ If the reporting bias is identified, we will explore possible reasons using the subgroup analysis or meta-regression analysis. $^{32}$

\section{DATA SYNTHESIS}

We will perform the meta-analysis when more than one trial examines the same intervention and outcomes with comparable methods in similar populations. If the statistical heterogeneity is not identified, the fixed-effect model will be built to estimate the overall intervention effects. ${ }^{32}$ Otherwise, the random-effect model will be used to provide more conservative results. ${ }^{32}$ When multiple intervention groups are used in a study, we will make pairwise comparisons by combining groups if possible. ${ }^{32}$ All statistical analyses will be performed by the RevMan V.5.3 software. The statistical significance is defined as $\mathrm{p}<0.05$. If the meta-analysis is not feasible, we will provide a narrative description of the results.

\section{Subgroup analysis and investigation of heterogeneity}

If possible, subgroup analyses will be conducted based on the following variables:

1. History of prenatal constipation.

2. Frequency of delivery.

3. Mode of delivery (vaginal delivery or caesarean section).

4. History of gastrointestinal diseases.

5. Type of comparisons.

6. Type of preparations (such as decoction, granula, ointment and capsule).
7. Different diagnostic criteria of constipation (Rome II/ III diagnostic criteria, clinical guidelines or defined by trialists).

8. Language or publication date.

9. The aetiology of postpartum constipation (pelvic floor injury, taking painkillers, a lack of adequate dietary fibre, vegetable, fruit and water, irregular meals, etc).

The difference of intervention effects across subgroups will be compared by $\chi^{2}$ test with $\mathrm{p}<0.05$ indicating statistical significance.

\section{SENSITIVITY ANALYSIS}

We will investigate the robustness of the pooled effects using sensitivity analyses according to the following variables if possible:

1. Impact of sample size: removing one or two studies in which sample size is more than $80 \%$ of participants in a meta-analysis. ${ }^{37}$

2. Impact of high risk of bias: removing studies in which overall risk of bias is high.

3. Impact of selected models: fixed-effect models versus random-effect models.

4. Impact of missing data: extreme worst-case analysis and best-case analysis. ${ }^{37}$

\section{Summary of findings tables}

Two review authors (JiZ and YL) will evaluate the quality of evidence for each outcome using the Grading of Recommendations Assessment, Development and Evaluation system. ${ }^{30}$ It will be categorised as high, moderate, low or very low. ${ }^{30}$ We will present the findings with a 'summary of finding' table. It will include all important outcomes, absolute and relative magnitude of effects, number of participants and a grade of the overall quality of the body of evidence for each outcome. ${ }^{30}$ Any discrepancy will be resolved by discussion or a consultation of a third review author (JuZ).

\section{Patient and public involvement}

Patients and public were not involved in development of the research question and outcome measures, the design of this study or the recruitment to and conduct of the study. There are no plans to disseminate the results to study participants. The burden of the intervention was not assessed by patients themselves for RCTs.

\section{Amendments}

If the protocol is modified, the change, the rationale and the date of any amendment will be described in the final report.

\section{Ethics and dissemination}

The final report of this systematic review will be published in a peer-reviewed scientific journal, and dataset will be made freely available.

\section{DISCUSSION}

This systematic review will provide a comprehensive review of the efficacy and safety of CHM for postpartum 
constipation. The evidence from this review may benefit patients with postpartum constipation and clinicians. It will also contribute to the development of relevant clinical guidelines. However, a large degree of heterogeneity in terms of methodological quality and outcome measures will likely pose challenges for study comparisons.

Contributors JZ, JS and JZ conceived the study. JZ, JS and JZ provided general guidance to the drafting of the protocol. JZ and YL drafted the protocol. JS designed the search strategy. JZ, YL, JL, SD, JS and JuZ drafted the manuscript. JZ, YL, JL, SD, JS and JZ reviewed and revised the manuscript. All authors have read and approved the final version of the manuscript.

Funding This study is supported by the Tianjin youth top talent project 2015 (lead by Junhua Zhang) and the National Natural Science Foundation of China (grant number 81703936).

Competing interests None declared.

Patient consent Not required.

Ethics approval No ethical issues are foreseen because no primary data will be collected.

Provenance and peer review Not commissioned; externally peer reviewed.

Open access This is an open access article distributed in accordance with the Creative Commons Attribution Non Commercial (CC BY-NC 4.0) license, which permits others to distribute, remix, adapt, build upon this work non-commercially, and license their derivative works on different terms, provided the original work is properly cited, appropriate credit is given, any changes made indicated, and the use is non-commercial. See: http://creativecommons.org/licenses/by-nc/4.0/.

\section{REFERENCES}

1. Turawa EB, Musekiwa A, Rohwer AC. Interventions for preventing postpartum constipation. Cochrane Database Syst Rev 2015;9:CD011625.

2. Bradley CS, Kennedy CM, Turcea AM, et al. Constipation in pregnancy: prevalence, symptoms, and risk factors. Obstet Gynecol 2007;110:1351-7.

3. Yang R, Hao J, Yu Y, et al. Postpartum defecation situation of women and influencing factors in central and western regions of Inner Mongolia. China Journal of Modern Medicine 2017;27:76-9.

4. Shin GH, Toto EL, Schey R. Pregnancy and postpartum bowel changes: constipation and fecal incontinence. Am J Gastroenterol 2015;110:521-9.

5. Corby H, Donnelly VS, O'Herlihy C, et al. Anal canal pressures are low in women with postpartum anal fissure. Br J Surg 1997;84:86-8.

6. Derbyshire E, Davies J, Costarelli V, et al. Diet, physical inactivity and the prevalence of constipation throughout and after pregnancy. Matern Child Nutr 2006;2:127-34.

7. Zhou Y, Yang X, Fan L, et al. Observations on the curative effect of lactulose for postpartum constipation based on a large sample study. Int J Clin Exp Med 2015;8:19167-71.

8. Technical Working Group, World Health Organization. Postpartum care of the mother and newborn: a practical guide. Birth 1999;26:255-8.

9. Chiarelli P, Cockburn J. The development of a physiotherapy continence promotion program using a customer focus. Aust $J$ Physiother 1999;45:111-9.

10. Shelton MG. Standardized senna in the management of constipation in the puerperium: a clinical trial. S Afr Med $J$ 1980;57:78-80.

11. Serra J, Mascort-Roca J, Marzo-Castillejo M, et al. Clinical practice guidelines for the management of constipation in adults. Part 1: Definition, aetiology and clinical manifestations. Gastroenterol Hepatol 2017;40:132-41.

12. Serra J, Mascort-Roca J, Marzo-Castillejo M, et al. Clinical practice guidelines for the management of constipation in adults. Part 2: Diagnosis and treatment. Gastroenterol Hepatol 2017;40:303-16.
13. Liu ZL, Li GQ, Bensoussan A, et al. Chinese herbal medicines for hypertriglyceridaemia. Cochrane Database Syst Rev 2013;6:CD009560.

14. Hu J, Zhang J, Zhao W, et al. Cochrane systematic reviews of Chinese herbal medicines: an overview. PLoS One 2011;6:e28696.

15. Zhu X, Liew Y, Liu ZL. Chinese herbal medicine for menopausal symptoms. Cochrane Database Syst Rev 2016;3:CD009023.

16. Xiong X, Li X, Zhang Y, et al. Chinese herbal medicine for resistant hypertension: a systematic review. BMJ Open 2015;5:e005355.

17. Cheng CW, Bian ZX, Zhu LX, et al. Efficacy of a Chinese herbal proprietary medicine (Hemp Seed Pill) for functional constipation. Am $J$ Gastroenterol 2011;106:120-9.

18. Chen CM, Lin LZ, Zhang EX. Standardized treatment of Chinese medicine decoction for cancer pain patients with opioid-induced constipation: a multi-center prospective randomized controlled study. Chin J Integr Med 2014;20:496-502.

19. Cheng CW, Bian ZX, Wu TX. Systematic review of Chinese herbal medicine for functional constipation. World J Gastroenterol 2009;15:4886-95.

20. Liu X. Xiaoyao powder for treating sixty-five patients with postpartum constipation. Gansu Journal of Traditional Chinese Medicine 2009;22:47.

21. Bo C, Li X. Chinese herbs enema for treating postpartum constipation. Yunnan Journal of Traditional Chinese Medicine 2014;35:48-9.

22. Turawa EB, Musekiwa A, Rohwer AC. Interventions for treating postpartum constipation. Cochrane Database Syst Rev 2014;9:CD010273.

23. Shamseer L, Moher D, Clarke M, et al. Preferred reporting items for systematic review and meta-analysis protocols (PRISMA-P) 2015: elaboration and explanation. BMJ 2015;350:g7647.

24. Xin HW, Fang XC, Zhu LM, et al. Diagnosis of functional constipation: agreement between Rome III and Rome II criteria and evaluation for the practicality. J Dig Dis 2014;15:314-20.

25. Cirillo C, Capasso R. Constipation and botanical medicines: an overview. Phytother Res 2015;29:1488-93.

26. Zheng $\mathrm{Q}$, Zheng $\mathrm{H}$, Lu L, et al. Acupuncture for functional constipation: protocol of an individual patient data meta-analysis. BMJ Open 2015;5:e007137.

27. Sajid MS, Hebbar M, Baig MK, et al. Use of prucalopride for chronic constipation: a systematic review and meta-analysis of published randomized, controlled trials. J Neurogastroenterol Motil 2016;22:412-22.

28. Chen M, Zheng H, Li J, et al. Non-pharmacological treatments for adult patients with functional constipation: a systematic review protocol. BMJ Open 2014;4:e004982.

29. Miller LE, Ibarra A, Ouwehand AC, et al. Normative values for stool frequency and form using Rome III diagnostic criteria for functional constipation in adults: systematic review with meta-analysis. Ann Gastroenterol 2017;30:161-7.

30. Hill PD, Aldag JC, Hekel B, et al. Maternal postpartum quality of life questionnaire. J Nurs Meas 2006;14:205-20.

31. Liberati A, Altman DG, Tetzlaff J, et al. The PRISMA statement for reporting systematic reviews and meta-analyses of studies that evaluate health care interventions: explanation and elaboration. PLOS Med 2009;6:e1000100.

32. Chandler J, Higgins JPT, Deeks JJ, et al. Cochrane handbook for systematic reviews of interventions version 5.2.0: Cochrane Community, 2017.

33. Aamann L, Dam G, Rinnov A, et al. Physical exercise for people with cirrhosis. Cochrane Database Syst Rev 2017;6:CD012678.

34. Weng H, Zeng XT, Li S, et al. Intrafascial versus interfascial nerve sparing in radical prostatectomy for localized prostate cancer: a systematic review and meta-analysis. Sci Rep 2017;7:11454.

35. Boonstra A, van Dulmen-den Broeder E, Rovers MM, et al. Severe fatigue in childhood cancer survivors. Cochrane Database Syst Rev 2017;6:CD012681.

36. Baicus C, Purcarea A, Elm Evon, et al. Alpha-lipoic acid for diabetic peripheral neuropathy. Cochrane Database Syst Rev 2018;2:CD012967.

37. Barbateskovic M, Schjorring OL, Jakobsen JC, et al. Higher versus lower inspiratory oxygen fraction or targets of arterial oxygenation for adult intensive care patients. Cochrane Database Syst Rev 2017;4:CD012631. 